\title{
Mucin Degradation in Human Colon Ecosystems
}

\author{
Isolation and Properties of Fecal Strains that Degrade ABH Blood Group \\ Antigens and Oligosaccharides from Mucin Glycoproteins
}

\author{
Lansing C. Hoskins, Manuel Agustines, William B. McKee, Erwin T. Boulding, Maria Kriaris, and George Niedermeyer \\ Gastroenterology Section, Department of Medicine, Veterans Administration Medical Center and \\ Case Western Reserve University School of Medicine, Cleveland, Ohio 44106
}

\begin{abstract}
We previously reported that the oligosaccharide chains of hog gastric mucin were degraded by unidentified subpopulations numbering $\sim 1 \%$ of normal human fecal bacteria. Here we report on the enzyme-producing properties of five strains of mucin oligosaccharide chain-degrading bacteria isolated from feces of four healthy subjects. Four were isolated from the greatest fecal dilutions yielding mucin side chain-degrading activity in culture, and thus were the numerically dominant side chain-degrading bacteria in their respective hosts. Three were Ruminococcus strains and two were Bifidobacterium strains. Two Ruminococcus torques strains, IX-70 and VIII239, produced blood group A- and H-degrading $\alpha$-glycosidase activities, sialidase, and the requisite $\beta$-glycosidases; these strains released $>90 \%$ of the anthrone-reacting hexoses from hog gastric mucin during growth in culture. The Bifidobacterium strains lacked A-degrading activity but were otherwise similar; these released $60-80 \%$ of the anthrone-reacting hexoses but not the $A$ antigenic structures from hog gastric mucin. Only Ruminococcus $A B$ strain VI-268 produced blood group B-degrading $\alpha$-galactosidase activity, but this strain lacked $\beta$ $N$-acetylhexosaminidases to complete degradation of $B$ antigenic chains. When this strain was co-cultured with a strain that produced $\boldsymbol{\beta}-\boldsymbol{N}$-acetylhexosaminidases, release of hexoses from blood group $B$ salivary glycoprotein increased from 50 to $>90 \%$, and bacterial growth was enhanced. The glycosidases required for side chain degradation were produced by these strains in the absence of mucin substrate, and a substantial fraction of each activity in stationary phase cultures was extracellular. In contrast, none of $\mathbf{1 6}$ other fecal Bacteroides, Escherichia coli, Streptococcus faecalis, and Bifidobacterium strains produced ABH blood group-degrading enzymes; other glycosidases produced by these strains were predominantly cell bound except for extracellular $\boldsymbol{\beta}-\boldsymbol{N}$-acetylhexosaminidases produced by the five $S$. faecalis strains.

We conclude that certain Bifidobacterium and Ruminococcus strains are numerically dominant populations degrading mucin oligosaccharides in the human colon due to their constitutive production of the requisite extracellular glycosidases including blood group antigen-specific $\alpha$-glycosidases. These properties characterize them as a functionally distinct subpopulation of normal human enteric microflora comprised of
\end{abstract}

An abstract of portions of this work was published in Gastroenterology 1983; 84:1191.

Received for publication 5 January 1984 and in revised form 23 November 1984.

J. Clin. Invest.

(C) The American Society for Clinical Investigation, Inc. 0021-9738/85/03/944/10 \$1.00

Volume 75, March 1985, 944-953 specialized subsets that produce blood group $H$ antigen-degrading glycosidases alone or together with either blood group A- or B-degrading glycosidases.

\section{Introduction}

There is mounting evidence that the adherent mucous gel layer of the gastrointestinal mucosa has important physiological roles as a lubricant (1), a protective barrier against mixing (2), a stabilizer for the mucosal association of some enteric bacteria, and a barrier to the association of others (3-5). Degradation of the gel layer would result in impairment of these functions. The large number of oligosaccharide side chains of mucin glycoproteins impart the characteristic hydrophilic and viscoelastic properties to the mucous gel $(1,6)$. In porcine and human gastric mucins, these chains are closely related linear and branched structures of varying length linked via $\alpha-N$ acetylgalactosamine to serine and threonine residues in the core polypeptide (7-10). A key feature is that the outer, nonreducing end of completed chains terminates in alphalinked glycosides while the inner regions are beta-glycoside sequences of D-galactose and $N$-acetyl-D-glucosamine. The terminal alpha-linked glycosides include sialic acids and the ABH blood group antigens whose antigenic specificity is conferred respectively by $\alpha-(1-3)-N$-acetyl-D-galactosamine (A), $\alpha$-(1-3)-D-galactose (B), and $\alpha$-(1,2)-L-fucose (H) linked to $\beta$ D-galactose.

Enteric bacterial communities degrade gut mucin glycoproteins in vivo (11-13) and in anaerobic fecal cultures (14-16), but the bacteria responsible for degrading mucin oligosaccharides have not been well characterized. Extensive degradation would require that the bacteria produce sialidase, the blood group $\mathrm{ABH}$-degrading $\alpha$-glycosidases, $\beta$-D-galactosidase, and $\beta$ - $N$-acetylglucosaminidase. Previous work from this laboratory indicated that the major degradation of the oligosaccharide chains of hog gastric mucin (HGM) ${ }^{1}$ in anaerobic cultures of human feces was by unidentified subpopulations averaging $1 \%$ of the fecal bacterial population (15). Chain degradation appeared to be dependent upon production of extracellular but not cell-bound sialidase, $\beta$-galactosidase, and $\beta$ - $N$-acetylglucosaminidase (16). Results of earlier studies indicated that extracellular $\alpha$-glycosidases capable of degrading the $\mathrm{ABH}$ blood group antigens were also produced by unidentified subpopulations of normal human fecal bacteria $(17,18)$.

We here report on the isolation and properties of five strains of human fecal bacteria that degrade mucin oligosaccharide chains and compare their glycosidase production with that of other enteric bacteria. We also describe a terminal

1. Abbreviations used in this paper: ACES buffer, N-(2-acetamido)-2aminoethanesulfonic acid; BGD, blood group-degrading; BSG, blood group B salivary glycoproteins; HGM, hog gastric mucins. 
dilution, replicate sampling method with fecal cultures that permitted us to determine the odds that the largest fecal population degrading HGM oligosaccharides represented a single strain that produced at least three of the requisite glycosidases rather than cooperation among different strains, each producing a single glycosidase. Use of this sampling design greatly facilitated isolation of three of the strains. The results indicate that these strains are members of a functionally distinct subset of fecal bacteria that degrades mucin oligosaccharides in man by producing extracellular glycosidases with the requisite linkage specificities.

\section{Methods}

Materials. Fresh stool samples were obtained from healthy subjects who had not received antibiotics in the preceding $3 \mathrm{mo}$. Stool samples from four randomly selected hospitalized patients were also used for isolation of Escherichia coli and Streptococcus faecalis strains.

Mucin substrates. Hog gastric mucin containing blood group A and $\mathrm{H}$ antigens at minimal hemagglutination-inhibiting concentrations of $80 \mathrm{ng} / \mathrm{mg}$ and $320 \mathrm{ng} / \mathrm{mg}$ lyophilized weight, respectively, was partially purified from a commercial source (lot 6901 , ICN Nutritional Biochemicals, Cleveland, $\mathrm{OH}$ ) as described previously (15). Blood group B salivary glycoproteins (BSG) were prepared from saliva from a blood group B secretor that was heated $10 \mathrm{~min}$ at $100^{\circ} \mathrm{C}$ and centrifuged at $700 \mathrm{~g}$. Ethanol was added to the supernate to $60 \% \mathrm{vol} /$ vol at $0-4^{\circ} \mathrm{C}$. The precipitated proteins were dissolved in $0.1 \mathrm{M} \mathrm{NaCl}$ containing $20 \mathrm{mM}$ Tris- $\mathrm{HCl}$ buffer, $\mathrm{pH} 7.8,5 \mathrm{mM} \mathrm{CaCl}$, and $3 \mathrm{mM}$ sodium azide. Pronase, amounting to $1 \%$ by weight of the BSG protein content, was added, and the mixture was incubated for $24 \mathrm{~h}$. A second, equal amount of pronase was then added and incubation carried out for an additional $24 \mathrm{~h}$. After centrifugation at $700 \mathrm{~g}$ the supernate was treated with ethanol to $60 \% \mathrm{vol} / \mathrm{vol}$. The resulting precipitate was dissolved in distilled water and heated $10 \mathrm{~min}$ at $100^{\circ} \mathrm{C}$ to inactivate pronase. It was then dialyzed against four changes of distilled water and was lyophilized. The yield of a typical preparation was $35 \mathrm{mg}$ from $100 \mathrm{ml}$ saliva; it contained $44 \%$ hexoses, $16 \%$ protein, and a minimal inhibitory concentration of B antigen of $325 \mathrm{ng} / \mathrm{mg}$ lyophilized weight. Partially deglycosylated HGM was prepared by adjusting a 10$\mathrm{mg} / \mathrm{ml} \mathrm{HGM}$ solution to $\mathrm{pH} 1.8$ with $\mathrm{HCl}$, refluxing for $8 \mathrm{~h}$ at $100^{\circ} \mathrm{C}$ and reprecipitating the mucin in $80 \% \mathrm{vol} / \mathrm{vol}$ ethanol after readjusting the $\mathrm{pH}$ to 7 . This mild acid hydrolysis cleaves the $\mathrm{A}$ and $\mathrm{H}$ antigenic determinant glycosides from the oligosaccharide chains, exposing the underlying core $\beta$-glycoside linkages (reference 19 and unpublished studies).

Unless stated otherwise, the anaerobic culture medium was prereduced "Medium 75" (15) containing $1 \mathrm{mg} / \mathrm{ml}$ glucose and $2 \mathrm{mg} / \mathrm{ml}$ HGM or BSG; it was employed either in liquid form or with $2 \% \mathrm{wt} /$ vol agar in roller tubes. Medium 75 containing $2 \mathrm{mg} / \mathrm{ml} \mathrm{D-glucose}$ and no HGM was used for comparing glycosidase production in the presence or absence of HGM. Specific culture media employed in the taxonomic identification of isolates were as listed in the VPI anaerobe laboratory manual (20). Azoalbumin and para-nitrophenyl-glycopyranoside substrates were obtained from Sigma Chemical Co., St. Louis, MO, and Calbiochem-Behring Corp., San Diego, CA. All chemicals were reagent grade.

\section{Methods}

Anaerobic culture techniques. The anaerobic roller tube technique (20) was used for propagation on agar-based solid media. Medium preparation, storage, inoculation, and incubation were performed in an atmosphere of $94 \% \mathrm{~N}_{2}-5 \% \mathrm{CO}_{2}-1 \% \mathrm{H}_{2}$ that had been passed over copper at $450^{\circ} \mathrm{C}$ to remove traces of oxygen.

Isolation techniques. The approach used in isolating fecal bacterial strains with mucin side chain degrading activity consisted of choosing the highest possible fecal dilutions so as to minimize overgrowth by facultative anaerobes and other species; using Medium 75 with $2 \mathrm{mg}$ / ml HGM but no glucose as a selection medium in which mucin oligosaccharide side chains were the sole source of carbohydrate; and screening single colonies growing on this medium for ability to produce A-, B-, or H-blood group antigen-degrading activity. Fresh feces was serially diluted in the medium and replicate roller tube cultures were inoculated from each of several fecal dilutions. After $48 \mathrm{~h}$ incubation, individual colonies were picked and screened for presence of blood group-degrading (BGD) activity. The blood group B-degrading Ruminococcus strain VI-268 was isolated from a heavily inoculated fecal culture containing $100 \mu \mathrm{g} / \mathrm{ml}$ neomycin sulfate to suppress facultative anaerobes. The other strains were each isolated from single cultures among replicates inoculated at the limiting fecal dilution where mucin degrading bacteria had been nearly diluted out. Colonies with BGD activity were restreaked onto roller tubes, and individual colonies were again picked and tested. Repetition of this process resulted in eventual isolation of pure strains. In order to enhance the likelihood that the isolates were pure cultures, a theoretical single clone was obtained from each by a terminal dilution method (21). The nominal probability that the growth represented a single clone varied from 76 to $95 \%$.

Five Bacteroides species, each from a different subject, were isolated from cultures inoculated with $0.4-1.0 \times 10^{-10} \mathrm{~g}$ feces. These were $B$. distasonis (1), B. ruminocola ss ruminocola (1), B. fragilis (1), and $B$. vulgatus (2). A single strain of Bifidobacterium longum was also isolated from one of the $10^{-10} \mathrm{~g}$ fecal inocula.

Five $E$. coli strains, each isolated from a different subject, and five $S$. faecalis strains, each also isolated from a different subject, were identified by standard confirmatory tests. Taxonomic identification of isolated anaerobic strains was made by comparison with results of published methods $(21,22)$. Identification of the five mucin side chaindegrading strains was made by the staff of the Anaerobe Laboratory, Virginia Polytechnic Institute, Blacksburg, VA. Guinea pig pathogenicity and mouse toxicity tests were performed as described (21).

Measurement of mucin degradation and enzyme production in in vitro cultures. Each strain was inoculated into triplicate $10-\mathrm{ml}$ tubes of Medium 75. Cultures were incubated anaerobically at $37^{\circ} \mathrm{C}$. Bacterial growth was monitored by measuring optical turbidity at $660 \mathrm{~nm}$. Bacterial cells were harvested after the onset of stationary growth phase at 22-71 h. Each culture was centrifuged for $20 \mathrm{~min}$ at $10,000 \mathrm{~g}$ to yield a cell-free supernatant fraction and a bacterial pellet fraction. A 2-ml aliquot of each supernate was heated for $5 \mathrm{~min}$ at $100^{\circ} \mathrm{C}$ to inactivate enzymes and was assayed for mucin degradation by measuring the residual $60 \% \mathrm{vol} / \mathrm{vol}$ ethanol precipitable hexose, protein, and $\mathrm{ABH}$ blood group antigen titers as previously described (15). Loss of $>25 \%$ of the anthrone-reacting hexoses from the $60 \%$ ethanol precipitable mucin was defined as indicating degradation of oligosaccharide chains (15). Results were expressed as the median and range of percentage loss of mucin hexoses among the triplicate cultures. The remaining supernate fraction and the bacterial pellets of each strain's triplicate cultures were pooled. The pooled supernate was dialyzed against 3-4 1-liter vol of $0.1 \mathrm{M} \mathrm{NaCl}$ containing $20 \mathrm{mM} \mathrm{N}$-(2-acetamido)-2aminoethanesulfonic acid (ACES buffer), pH 6.4, $1 \mathrm{mM} \mathrm{MgCl}_{2}$, and $0.1 \mathrm{mM} \mathrm{Na} 2$ EDTA ("enzyme assay buffer"). The pooled bacterial pellets were washed once and disrupted by ultrasonication for $4 \mathrm{~min}$ as previously described (16). The disrupted cell suspension was centrifuged for $30 \mathrm{~min}$ at $12,000 \mathrm{~g}$, yielding a pellet of bacterial cell residue and a soluble bacterial sonicate fraction. The soluble sonicate fraction was then dialyzed against 3-4 1-liter vol of enzyme assay buffer.

Enzyme assays were performed as previously described $(16,17)$ on the culture supernate and on the bacterial sonicate fraction of each culture representing, respectively, extracellular and cell-bound enzyme activity. Blood group A-, B-, and $\mathrm{H}$-antigen degrading activities were measured by the rate of decrease of the hemagglutination inhibition titer of each antigen substrate during incubation with culture fractions. Substrates were HGM for A and $\mathrm{H}$ antigen and $B$ salivary glycoproteins for B antigen. Sialidase was measured by the rate of cleavage of sialic acid from human serum orosomucoid. $\beta$-glucuronidase was assayed using phenolphthalein glucuronide as substrate (23). All other glycosidase activities were measured by the rate of cleavage of para-nitrophenol 
from their corresponding p-nitrophenyl-glycopyranosides. Protease activity was measured using $25 \mathrm{mg} / \mathrm{ml}$ azoalbumin in $0.1 \mathrm{M}$ Tris- $\mathrm{HCl}$

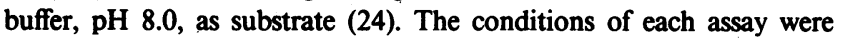
chosen so that rate of substrate loss or product formation was proportional to enzyme concentration. $1 \mathrm{U}$ of BGD activity was defined as that amount of activity that caused the titer of antigen substrate to decrease by one twofold dilution in $30 \mathrm{~min}$ at $37^{\circ} \mathrm{C}$. Inactive preparations were defined as those causing no decrease in antigen titer after incubation for $24 \mathrm{~h} .1 \mathrm{mU}$ of the other glycosidases was that amount releasing $1 \mathrm{nmol}$ of product per minute. $1 \mathrm{U}$ of protease activity was defined as that amount releasing trichloracetic acid-soluble products with an optical density of 0.001 at $440 \mathrm{~nm}$ after $24 \mathrm{~h}$ incubation. Activities were expressed as the sum of extracellular and cell-bound units per milligram of bacterial protein. Sialidase, $p$-nitrophenylglycosidase, and $\beta$-glucuronidase activities listed as zero in the tables were those which were $\leq 0.5 \mathrm{mU} / \mathrm{mg}$ bacterial protein. The coefficients of variation for five glycosidase activities produced in triplicate cultures of two mucin-degrading strains and one Bacteroides strain averaged 15\% (range: $3-26 \%$ ).

The ability of the extracellular $\beta$ - $N$-acetyl hexosaminidases produced by the $S$. faecalis strains and others to cleave $N$-acetylhexosamines from mucin oligosaccharides was assessed by measuring the amount of $N$-acetylhexosamines released from a $10-\mathrm{mg} / \mathrm{ml}$ solution of partially deglycosylated mucin in enzyme assay buffer during incubation with an equal volume of the cell-free culture supernate at $37^{\circ} \mathrm{C}$. Aliquots were removed from the incubation mixture initially and at $24 \mathrm{~h}$, and the mucin was precipitated from them with $80 \% \mathrm{vol} / \mathrm{vol}$ ethanol. After centrifugation, the supernatant fractions were evaporated to dryness, redissolved in water, and assayed for released $N$-acetylhexosamines by the method of Reissig et al, (25) using $\mathrm{N}$-acetylglucosamine as standard.

Protein concentration in the bacterial cell sonicate and the cell residue fractions was determined by a modification (15) of the method of Lowry et al. (26). Since ACES buffer formed a chromogen with the Folin phenol reagent protein, assays were performed on aliquots of the cell sonicate fractions that had been dialyzed against enzyme assay

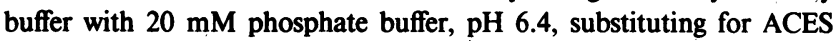
buffer. Bacterial protein concentration was the sum of the protein concentrations of the cell sonicate and cell residue. ABO blood group and secretor status of subjects were determined using standard hemagglutination techniques as previously described (17).

Terminal dilution, replicate sampling method for testing the single strain hypothesis

General features. Fresh feces was diluted to the point where bacteria degrading mucin side chains were present in some, but not in all, cultures inoculated from this dilution. Eight or 12 replicate tubes of anaerobic culture medium were inoculated from this dilution, and after $48 \mathrm{~h}$ incubation the cell-free supernates of every culture were analyzed for mucin side chain degradation and for four glycosidase activities: $\beta$-D-galactosidase (A), $\beta$ - $N$-acetyl-D-glucosaminidase (B), blood group H-degrading activity (C), and blood group A-degrading activity (D). Hypothesis I states that the glycosidases are produced by a single strain. Under this hypothesis the glycosidases would occur together only in those cultures containing the single strain, and only in those cultures would there be extensive degradation of mucin side chains. Hypothesis II states that each glycosidase is produced by a different strain. Under this hypothesis each glycosidase would be randomly and independently distributed among the replicate cultures, and extensive side chain degradation would only occur in those cultures where by chance all four glycosidases were present together. Each hypothesi. carries with it a different probability of finding cultures containing all glycosidases. These probabilities were calculated using binomial probability Eqs. 1 and 2, listed in the Appendix, and the ratio of their probabilities represented the odds favoring hypothesis I over hypothesis II.

It was possible to estimate the appropriate fecal inoculum size by taking advantage of two properties of fecal populations of mucindegrading bacteria (15): their fecal population densities are generally stable and the frequency of cultures containing these bacteria among replicate cultures inoculated with serially diluted feces follows a Poisson distribution according to equation (3):

$y=n\left(1-e^{-\mathrm{od}}\right)$,

where $y$ equals number of cultures with mucin side chain degradation; $n$ equals number of inoculated replicate cultures; $d$ equals most probable number of mucin side chain-degrading bacteria per gram wet fecal weight; and $v$ equals fecal inoculum size, $g$. When $y=n / 2$, Eq. 3 simplifies to:

$v=0.693 / d$.

Accordingly, the appropriate fecal inoculum size was estimated by first determining the most probable number of mucin side chain-degrading bacteria per gram in a preliminary fecal sample (15) and substituting this value for $d$ in Eq. 4 to obtain the fecal inoculum size estimated to yield side chain-degrading bacteria in one-half of the replicate cultures. Using this value for $v$ as a gijide we inoculated three sets of replicate cultures within a 10 -fold size range, two with less than $v$, and one with greater than $v$. After incubation, the cell-free culture supernates from the three sets were screened for mucin hexose degradation, and that set was chosen for further analysis that displayed strong mucin hexose degradation in less than half of the cultures.

Loss of $>70 \%$ of anthrone-reacting hexoses from HGM was defined as extensive side chain degradation. Presence of blood group A- or H-degrading activity was detected by a decrease of blood group A or $H$ hemagglutination inhibition titers to $\leq 1: 2$ from their initial titers in uninoculated medium; no change in the $\mathrm{A}$ or $\mathrm{H}$ titers indicated lack of activity. $\beta$-galactosidase and $\beta$ - $N$-acetylglucosaminidase were defined as present if their activities were $\geq 2.0 \mathrm{mU} / \mathrm{ml}$ and $\geq 1.0 \mathrm{mU} / \mathrm{ml}$, respectively. Use of these cutoff values eliminated low levels of background activity in cultures with no loss of mucin hexoses and discriminated between such cultures and those with loss of $>70 \%$ anthrone-reacting mucin hexoses, the majority of which had activities exceeding $5 \mathrm{mU} / \mathrm{ml}$.

\section{Results}

Before attempting to isolate fecal bacteria that degraded HGM oligosaccharides it was important to determine the likelihood that the largest fecal population degrading these oligosaccharides was a single bacterial strain that produced the requisite glycosidases, or if it represented different strains, each of which produced a single glycosidase, acting cooperatively to effect chain degradation. To this end we used a terminal dilution, replicate sampling method to calculate the odds that four requisite glycosidases in anaerobic fecal cultures were produced by a single strain. Replicate cultures from seven subjects were inoculated at the limiting fecal dilution where bacteria degrading mucin oligosaccharides were still present in some of the replicates but not in others. Glycosidase activities were variably distributed among these cultures; the distribution pattern varied with fecal inoculum size and from subject to subject. Extensive loss (>70\%) of HGM hexoses occurred in $95 \%$ of cultures containing either three or four of the glycosidases, and was greatest in those containing all four glycosidases (median loss of mucin hexoses $=95 \%$, range $=79-99 \%$ ).

Table I shows the distribution of glycosidases among replicate fecal cultures from each of the subjects. This table also lists the probabilities under hypotheses I (a single strain produces every glycosidase) and II (each glycosidase is produced by a different strain) for obtaining the observed results and the odds favoring hypothesis I. The odds favoring hypothesis I exceeded 150 to 1 in four subjects and were $<10$ to 1 in three. These odds sufficiently favored the single strain hypothesis that we used production of A- or H-degrading activity as a 
Table I. Probabilities and Odds Associated with Hypothesis $I^{*}$ and Hypothesis II

\begin{tabular}{|c|c|c|c|c|c|c|c|c|c|c|}
\hline \multirow[b]{2}{*}{ Subject } & \multirow{2}{*}{$\begin{array}{l}\text { Fecal } \\
\text { inoculum }\end{array}$} & \multirow{2}{*}{$\begin{array}{l}\text { No. of } \\
\text { replicate } \\
\text { cultures }\end{array}$} & \multicolumn{4}{|c|}{$\begin{array}{l}\text { No. of cultures containing } \\
\text { glycosidase§ }\end{array}$} & \multirow{2}{*}{$\begin{array}{l}\text { No. of cultures } \\
\text { containing } \\
\text { every glycosidase }\end{array}$} & \multicolumn{2}{|c|}{ Probability with hypothesis } & \multirow{2}{*}{$\begin{array}{l}\text { Odds favorin } \\
\text { hypothesis I }\end{array}$} \\
\hline & & & A & B & $\mathrm{C}$ & D & & $I^{*}$ & II & \\
\hline & $g$ & $n$ & $a$ & $b$ & $c$ & $d$ & $r$ & & & \\
\hline 1 & $2 \times 10^{-10}$ & 12 & 4 & 4 & 4 & 0 & 4 & 0.238 & $6.89 \times 10^{-4}$ & $346 / 1$ \\
\hline 2 & $3 \times 10^{-10}$ & 12 & 2 & 1 & 2 & 1 & 1 & 0.384 & $2.31 \times 10^{-3}$ & $166 / 1$ \\
\hline 3 & $1 \times 10^{-8}$ & 8 & 2 & 3 & 3 & 2 & 2 & 0.312 & $2.05 \times 10^{-3}$ & $152 / 1$ \\
\hline 4 & $1 \times 10^{-9}$ & 8 & 3 & 3 & 2 & 2 & 2 & 0.312 & $2.05 \times 10^{-3}$ & $152 / 1$ \\
\hline 5 & $1 \times 10^{-9}$ & 8 & 7 & 4 & 3 & 2 & 2 & 0.312 & $3.66 \times 10^{-2}$ & $8.5 / 1$ \\
\hline 6 & $3 \times 10^{-10}$ & 12 & 6 & 3 & 4 & 2 & 1 & 0.384 & $7.72 \times 10^{-2}$ & $5.0 / 1$ \\
\hline 7 & $3 \times 10^{-10}$ & 12 & 10 & 8 & 2 & 1 & 1 & 0.384 & $8.50 \times 10^{-2}$ & $4.5 / 1$ \\
\hline
\end{tabular}

* A single strain produces all of the glycosidases. $¥$ Separate strains produce individual glycosidases. $\S \mathrm{A}, \beta$-D-galactosidase; B, $\beta$ - $N$-acetyl-Dglucosaminidase; C, blood group H-degrading activity; D, blood group A-degrading activity.

screening method to isolate representatives of these hypothetical single strains from selected replicate cultures. Three of the five strains of bacteria with side chain-degrading activity were isolated: Ruminococcus torques strain VIII-239 and Bifidobacterium strains VIII-210 and VIII-240. Consistent with hypothesis I, each produced three of the extracellular glycosidases, and $\boldsymbol{R}$. torques strain VIII-239 also produced A-degrading activity.

Characteristics of the isolated strains. The five strains with mucin oligosaccharide chain-degrading activity were non-sporulating, gram-positive, obligate anaerobes of the genera Ruminococcus and Bifidobacterium. Strains IX-70 and VIII-239 were identified as $R$. torques, strain VI-268 as $R$. $A B$, strain VIII-210 as Bifidobacterium bifidum, and strain VIII-240 as $B$. infantis. Some properties of the B-degrading enzyme produced by strain VI-268 were reported previously $(17,18)$. Like other members of these genera, growth of each was enhanced by carbohydrates including glucose which were fermented with production of acid. None were pathogenic to guinea pigs or toxigenic in the mouse assay. The $\mathrm{ABO}$ blood group and $\mathrm{ABH}$ secretor status of their original hosts and the concentration of fecal inoculum from which they were isolated are listed with each strain in Table II. $R$. $A B$ strain VI-268 and $R$. torques strain VIII-239 were isolated from one blood group B secretor; each of the others was isolated from a different subject. All but strain VI-268 were isolated from cultures at the limiting fecal dilution exhibiting mucin side chain degradation in culture and thus were the numerically dominant fecal bacteria with side chain-degrading activity in their respective hosts. Since the blood group A-degrading $R$. torques strain IX-70 was isolated from a blood group A secretor and the blood

Table II. Degradation of Mucin Oligosaccharides by Mucin Side Chain Degraders and Other Fecal Bacteria

\begin{tabular}{|c|c|c|c|c|c|c|c|c|}
\hline \multirow[b]{3}{*}{ Strain } & \multirow{3}{*}{$\begin{array}{l}\text { Host's blood } \\
\text { group and } \\
\text { secretor status }\end{array}$} & \multirow{3}{*}{$\begin{array}{l}\text { Fecal } \\
\text { concentration } \\
\text { from which } \\
\text { isolated }\end{array}$} & \multicolumn{2}{|c|}{$\begin{array}{l}\text { Median (and range) percent loss } \\
\text { of hexoses from: }\end{array}$} & \multicolumn{4}{|c|}{ Loss of blood group antigen titers from: } \\
\hline & & & \multirow[b]{2}{*}{ HGM } & \multirow[b]{2}{*}{ BSG } & \multicolumn{2}{|l|}{ HGM } & \multicolumn{2}{|l|}{ BSG } \\
\hline & & & & & $\mathbf{A}$ & $\mathbf{H}$ & B & $\mathbf{H}$ \\
\hline & & $\mathrm{g} / \mathrm{ml}$ & & & & & & \\
\hline \multicolumn{9}{|l|}{ I. Mucin chain degraders } \\
\hline \multicolumn{9}{|l|}{ Ruminococcus } \\
\hline torques strain IX-70 & A sec. & $1 \times 10^{-8}$ & $95(94-95)$ & $44(42-46)$ & Comp.* & Comp. & None & Comp. \\
\hline torques strain VIII-239 & B sec. & $1 \times 10^{-9}$ & $91(90-91)$ & $39(38-41)$ & Comp. & Comp. & None & Comp. \\
\hline$A B$ strain VI-268 & B sec. & $2 \times 10^{-5}$ & $34(34-36)$ & $50(48-52)$ & None & Comp. & Comp. & Comp. \\
\hline \multicolumn{9}{|l|}{ Bifidobacterium } \\
\hline bifidum strain VIII-210 & 0 sec. & $4 \times 10^{-11}$ & $81(80-81)$ & $50(48-53)$ & None & Comp. & None & Comp. \\
\hline infantis strain VIII-240 & 0 sec. & $1 \times 10^{-8}$ & $69(65-70)$ & $47(47-51)$ & None & Comp. & None & Comp. \\
\hline \multicolumn{9}{|l|}{ II. Other strains } \\
\hline \multicolumn{2}{|l|}{ Bacteroides species (5) } & $\leq 1 \times 10^{-10}$ & $25(20-27)$ & & & & & \\
\hline \multicolumn{2}{|c|}{ Bifidobacterium longum (1) } & $1 \times 10^{-10}$ & $22(20-24)$ & & & & & \\
\hline \multicolumn{2}{|l|}{ E. coli strains (5) } & $1 \times 10^{-5}$ & $19(9-23)$ & & & & & \\
\hline \multicolumn{2}{|l|}{ S. faecalis strains (5) } & $\sim 1 \times 10^{-3}$ & $21(19-23)$ & & & & & \\
\hline
\end{tabular}

* Comp., complete loss of antigen. 
group H-degrading Bifidobacterium strains VIII-210 and VIII240 were isolated from blood group $O$ secretors, each of these was also the numerically dominant strain degrading its host's blood group phenotype antigen in his gut mucous secretions.

Degradation of mucin side chains. Loss of hexoses and blood group antigens from HGM and BSG during growth of the five strains in culture is shown in Table II. Loss of hexoses from HGM by side chain-degrading strains varied from $>90 \%$ for $R$. torques strains IX-70 and VIII-239 to $34 \%$ for $R$. $A B$ strain VI-268. Loss of blood group antigens was either complete or nil. The $R$. torques strains completely degraded the A and $H$ antigen structures from the side chains. In contrast, the other three strains degraded $\mathrm{H}$ antigenic determinants but not A antigenic determinants, and loss of hexoses from HGM by these three strains was less extensive. Loss of hexoses from BSG by these five strains was limited to $39-50 \%$ and included complete loss of $\mathrm{H}$ antigenic determinants. Of the five strains, only $R$. $A B$ strain VI-268 degraded B antigen from BSG; this degradation was accompanied by a release of galactose from BSG (17).

Table II also shows the extent of mucin degradation by strains of Bacteroides species (5), B. longum (1), E. coli (5), and $S$. faecalis (5). Little or no degradation of hexoses occurred from HGM during growth of these strains. In only one out of 48 cultures did the extent of hexose degradation from HGM (27\%) exceed the defined lower limit of hexose degradation (26).

Glycosidase production by mucin side chain degraders.
Glycosidase activities produced by side chain-degrading bacteria during growth in culture are shown in Table III. The listed glycosidases are those expected to have a role in side chain degradation. Blood group A-degrading and $p$-nitrophenyl- $\alpha-N$ acetylgalactosaminidase activities are probably due to the same enzyme since we (unpublished studies with strain IX-70) and others (27) have found that these activities have the same electrophoretic mobility. The side chain-degraders differed in the specificity of the $\alpha$-glycosidases they produced. While all produced H-degrading activity and sialidase, only the two $R$. torques strains produced A-degrading activity and $\alpha-N$ acetylgalactosaminidase. $R$. $A B$ strain VI-268 was the only one that produced B-degrading activity. The two Bifidobacterium strains produced only $\mathrm{H}$-degrading activity. Each of the strains produced $\beta$-galactosidase, and all except $R$. $A B$ strain VI-268 produced the two $\beta$ - $N$-acetylhexosaminidases.

Two properties of glycosidase production by the side chaindegrading strains are noteworthy. First, a substantial fraction of their activities is extracellular. Thus, by early stationary growth phase the median percentage extracellular activity for the eight glycosidases among the five strains in Table III was $49 \%$ and ranged from 0 to $96 \%$. In general, the extracellular fraction of the glycosidase activities was greater among the three Ruminococcus strains (median $=67 \%$ ) than among the two Bifidobacterium strains (median $=22 \%$ ). Second, production of glycosidases required for mucin side chain degradation is constitutive, i.e., their synthesis is not induced by mucin.

Table III. Glycosidase Activities in Cultures of Mucin Side Chain Degraders and Other Fecal Bacteria*

\begin{tabular}{|c|c|c|c|c|c|c|c|c|}
\hline \multirow[b]{2}{*}{ Strain } & \multicolumn{3}{|c|}{ BGD activity vs. } & \multirow[b]{2}{*}{ Sialidase } & \multirow{2}{*}{$\begin{array}{l}\alpha-N(\text { Ac)galactos- } \\
\text { aminidase }\end{array}$} & \multirow[b]{2}{*}{$\beta$-Galactosidase } & \multirow{2}{*}{$\begin{array}{l}\beta-N(\text { Ac)glucos- } \\
\text { aminidase }\end{array}$} & \multirow{2}{*}{$\begin{array}{l}\beta-N(\text { Ac)galactos- } \\
\text { aminidase }\end{array}$} \\
\hline & $\mathbf{A}$ & B & $\mathbf{H}$ & & & & & \\
\hline \multirow{3}{*}{\multicolumn{9}{|c|}{$\begin{array}{l}\text { I. Mucine chain degraders } \\
\text { Ruminococcus } \\
\text { torques strain }\end{array}$}} \\
\hline & & & & & & & & \\
\hline & & & & & & & & \\
\hline IX-70 & $214(44 \%)$ & 0 & $12(33 \%)$ & $55(49 \%)$ & $460(41 \%)$ & $555(33 \%)$ & $360(53 \%)$ & $21(37 \%)$ \\
\hline \multicolumn{9}{|l|}{ torques strain } \\
\hline VIII-239 & $244(82 \%)$ & 0 & $25(89 \%)$ & $81(69 \%)$ & $490(75 \%)$ & $300(69 \%)$ & $240(73 \%)$ & $49(66 \%)$ \\
\hline$A B$ strain VI-268 & 0 & $1,100(86 \%)$ & $24(57 \%)$ & $6(67 \%)$ & 0 & $25(96 \%)$ & 0 & 0 \\
\hline \multicolumn{9}{|l|}{ Bifidobacterium } \\
\hline \multicolumn{9}{|l|}{ bifidum strain } \\
\hline VIII-210 & 0 & 0 & $31(50 \%)$ & $72(33 \%)$ & 0 & $2,800(10 \%)$ & $860(25 \%)$ & $17(8 \%)$ \\
\hline \multicolumn{9}{|l|}{ infantis strain } \\
\hline VIII-240 & 0 & 0 & $4(25 \%)$ & $4(48 \%)$ & 0 & $760(2 \%)$ & $110(18 \%)$ & $2(0 \%)$ \\
\hline \multicolumn{9}{|l|}{ II. Other strains } \\
\hline Bacteroides & 0 & 0 & 0 & $3.5(0 \%)$ & $0.1(0 \%)$ & $340(4.4 \%)$ & $1,300(0.6 \%)$ & $160(0 \%)$ \\
\hline species (5) & & & {$[0-0.5]$} & {$[0.4-11]$} & {$[0-1.0]$} & {$[140-840]$} & {$[230-1,700]$} & {$[45-240]$} \\
\hline \multicolumn{9}{|l|}{ Bifidobacterium } \\
\hline longum (1) & 0 & 0 & 0 & 0 & 0 & $300(0 \%)$ & $15(20 \%)$ & $1.6(0 \%)$ \\
\hline \multirow[t]{2}{*}{ E. coli strains (5) } & 0 & 0 & 0 & 0 & 0 & $3.0(14 \%)$ & 0 & $\mathbf{0}$ \\
\hline & & & & {$[0-1.0]$} & & {$[3.0-6.0]$} & & \\
\hline \multirow[t]{2}{*}{ S. faecalis strains (5) } & & 0 & 0 & 0 & 0 & 0 & $230(90 \%)$ & $9.0(90 \%)$ \\
\hline & & & & & & & [80-280] & {$[6.0-10]$} \\
\hline
\end{tabular}

\footnotetext{
* Values are for total (cell-bound plus extracellular) activity followed by percentage of extracellular activity in parentheses. Values for the five Bacteroides species, the five $E$. coli strains and the 5 Str. faecalis strains are the medians with the range in brackets underneath. Activities are expressed per milligram bacterial protein. $1 \mathrm{U}$ of A-, B- or H-blood group degrading activity is that amount causing the antigen titer to decrease by one twofold serial dilution in $30 \mathrm{~min}$. All other activities: milliunits (nanomole product released per minute).
} 
This is shown in Table IV, which expresses the ratio of cellbound plus extracellular glycosidase activities produced in medium containing HGM to the activities produced by simultaneously inoculated cultures in medium lacking HGM. If HGM induced synthesis of these activities the ratio should exceed 1.00 by many-fold; if not, the ratio should be one or less. The results show that with four of the isolates the ratio ranged from 0.3 to 1.9. Although the glycosidase activities of Bifidobacterium strain VIII-210 were enhanced threefold in medium containing HGM it is questionable if this represents induced synthesis, since with induction the ratio usually far exceeds 3.0 (28).

Glycosidase production by other fecal bacteria (Table III). In contrast to the mucin side chain-degrading bacteria, none of the other fecal isolates produced extracellular A-, B-, or Hblood group-degrading activity. Weak cell-bound $\mathrm{H}$-degrading activity was produced by one Bacteroides vulgatus strain and one $B$. fragilis strain that required prolonged incubation for $\geq 24 \mathrm{~h}$ to detect. The Bacteroides strains produced the other glycosidases in amounts that differed widely among strains. Highest activities of most glycosidases were produced by the two $B$. vulgatus strains and by the single $B$. fragilis strain. Glycosidases produced by the Bacteroides strains were almost exclusively cell bound. Thus, the median extracellular activity of $\mathbf{1 0}$ glycosidases produced by these strains varied from 0 to $4.4 \%$ of the total activity. As with the mucin side chaindegrading bacteria, the levels of glycosidase activities produced by the Bacteroides strains were uninfluenced by the presence of HGM in the culture medium (data not shown).

Among the eight glycosidase activities, only cell-bound $\beta$ galactosidase and $\beta-N$-acetylhexosaminidases were detected in cultures of the $B$. longum strain. Small amounts of cell-bound $\beta$-galactosidase were produced by the $E$. coli strains which increased many-fold when these strains were grown in medium containing lactose (data not shown).

All five $S$. faecalis strains produced the two $\beta$ - $N$-acetylhexosaminidase activities as extracellular enzymes. While active towards their $p$-nitrophenylglycoside substrates, these glycosidases did not release $N$-acetylhexosamines from oligosaccharide chains of partially deglycosylated mucin. Thus, cell-free culture supernates of each of the $S$. faecalis strains incubated with partially deglycosylated mucin for $24 \mathrm{~h}$ released $<1 \mathrm{nmol}$ of $\mathrm{N}$-acetylglucosamine/mg mucin substrate, whereas culture supernates of strains IX-70 and VIII-240 in parallel incubations released 145 and $205 \mathrm{nmol} / \mathrm{mg}$ mucin, respectively.
Relationship between degradation of mucin oligosaccharides and the glycosidases produced by fecal bacteria. When compared to other fecal strains, the ability of the five mucin side chaindegraders to degrade HGM oligosaccharides was clearly related to their production of extracellular glycosidase activities, particularly the $\alpha$-glycosidases that degraded the ABH blood group antigens. Thus, only $R$. torques strains IX-70 and VIII-239 produced all of the requisite glycosidases, including A-degrading activity. Only in cultures of these strains was there $\geq 90 \%$ loss of hexoses and complete loss of blood group $\mathrm{A}$ and $\mathrm{H}$ antigen from HGM side chains. By contrast, Bifidobacterium strains VIII-210 and VIII-240 produced the other glycosidases but not the A-degrading $\alpha$ - $N$-acetylgalactosaminidase; these strains degraded less (65-80\%) hexoses from HGM and caused complete loss of $\mathrm{H}$ antigen but no loss of $\mathrm{A}$ antigen. Ruminococcus strain VI-268 lacked $\beta$ - $N$-acetylhexosaminidases as well as Adegrading activity; this strain caused the least degradation of hexoses from HGM.

Limited degradation of side chains from BSG by the five side chain-degrading strains is consistent with the lack, in four strains, of blood group B-degrading activity for the initial step in the sequential stepwise cleavage of $B$ antigenic chains. Although the fifth strain, $R$. $A B$ VI-268, produced a Bdegrading $\alpha$-galactosidase, its lack of $\beta$-hexosaminidases may have prevented it from completing the degradation of BSG side chains. In order to test this possibility we co-cultured strain VI-268 with strain VIII-210, which produced $\beta-N$ acetylhexosaminidases, in medium containing BSG and compared side chain degradation and bacterial growth in the cocultures with cultures containing each strain alone. The results in two studies (Table V) showed that degradation of hexoses from BSG was increased from $40-50 \%$ to $>90 \%$ in the coculture, and bacterial growth was enhanced $\geq 3$-fold. Examination of gram-stained smears of co-cultures showed that roughly one-third of the bacteria were strain VIII-210, indicating that growth of both strains had been enhanced. When the living bacteria of strain VIII-210 were replaced by a sterile cell-free culture supernate of this strain (study 3 in Table V), loss of hexoses from BSG were doubled from 39 to $81 \%$ and growth of strain VI-268 increased fivefold, compared with cultures containing supernate preheated at $100^{\circ} \mathrm{C}$ for $5 \mathrm{~min}$ in order to inactivate its glycosidase activities. These results are consistent with an in vitro symbiotic cooperation between strains VI-268 and VIII-210, wherein each produced a glycosidase that the other lacked. When grown together the full

Table IV. Evidence for Constitutive Production of Glycosidase Activities by Mucin Side Chain Degrading Strains: Ratio of Activities in Cultures Containing HGM to Activities in Cultures without HGM*

\begin{tabular}{|c|c|c|c|c|c|c|}
\hline \multirow[b]{2}{*}{ Strain } & \multicolumn{3}{|c|}{ BGD activity vs. } & \multirow[b]{2}{*}{ Sialidase } & \multirow[b]{2}{*}{$\beta$-Galactosidase } & \multirow[b]{2}{*}{$\beta-N($ Ac)glucosaminidase } \\
\hline & A & B & $\mathbf{H}$ & & & \\
\hline \multicolumn{7}{|l|}{ Ruminococcus } \\
\hline torques strain IX-70 & 0.40 & & 1.00 & 0.32 & 0.73 & 0.30 \\
\hline torques strain VIII-239 & 0.69 & & 0.53 & 1.50 & 0.93 & 1.28 \\
\hline$A B$ strain VI-268 & & 0.78 & 0.75 & 1.93 & 0.43 & \\
\hline \multicolumn{7}{|l|}{ Bifidobacterium } \\
\hline bifidum strain VIII-210 & & & 3.00 & 3.50 & 3.60 & 2.47 \\
\hline infantis strain VIII-240 & & & 0.91 & 0.68 & 0.88 & 0.70 \\
\hline
\end{tabular}

* Activities are the total (cell-bound plus extracellular) glycosidase activities in each culture expressed as units per milligram bacterial protein. 
Table V. Cooperation Between Ruminococcus Strain VI-268 and Bifidobacterium Strain VIII-210 in the Degradation of Oligosaccharide Side Chains from Blood Group B Salivary Mucin Glycoproteins*

\begin{tabular}{|c|c|c|c|c|c|c|}
\hline \multirow[b]{2}{*}{ Strain } & \multicolumn{3}{|c|}{ Percentage loss of mucin hexoses } & \multicolumn{3}{|c|}{ Bacterial growth ( $\Delta$ optical density $660 \mathrm{~nm}$ ) } \\
\hline & Study 1 & Study 2 & Study 3 & Study 1 & Study 2 & Study 3 \\
\hline VI-268 alone & $50 \pm 2$ & $38 \pm 4$ & $41 \pm 3$ & $46 \pm 2$ & $38 \pm 3$ & $12 \pm 6$ \\
\hline VIII-210 alone & $50 \pm 3$ & $42 \pm 19$ & & $20 \pm 3$ & $23 \pm 12$ & \\
\hline VI-268 + VIII-210 co-culture & $97 \pm 1$ & $94 \pm 2$ & & $126 \pm 11$ & $152 \pm 31$ & \\
\hline \multicolumn{7}{|l|}{ VI-268 + heat-inactivated culture supernate } \\
\hline of VIII-210 & & & $39 \pm 2$ & & & $21 \pm 7$ \\
\hline VI-268 + culture supernate of VIII-210 & & & $81 \pm 1$ & & & $100 \pm 7$ \\
\hline
\end{tabular}

* Values are the mean $\pm \mathrm{SD}$ of triplicate cultures. The values for bacterial growth in the table are the observed values multiplied by 1,000 .

complement of glycosidases was produced that was required for extensive degradation of BSG oligosaccharides. Since bacterial growth in Medium 75 is limited by carbohydrate concentration (15), the greater bacterial growth in the co-cultures presumably resulted from the greater amount of saccharides released from BSG by cooperation between the two strains.

Production of seven other glycosidases was tested in cultures of each of the 21 isolates (Table VI). Weak extracellular $\alpha-N$ acetylglucosaminidase activity was detected only in cultures of $\boldsymbol{R}$. torques strains VIII-239 and IX-70. Inability of most strains to produce this activity is consistent with recent observations that oligosaccharide chains with $\alpha-N$-acetylglucosamine at their nonreducing end are not a feature of human gastric mucin (9) despite being prominent in porcine gastric mucin $(7,8)$. All mucin side chain degrading strains, Bacteroides strains, and the $B$. longum strain produced $\alpha$-glucosidase, and all of these except $R$. torques strains IX-70 and VIII-239 produced $\alpha$ galactosidase. The $p$-nitrophenyl- $\alpha$-galactosidase produced by strain VI-268 differed from its blood group B-degrading $\alpha$ galactosidase in having greater electrophoretic mobility, greater retardation during gel exclusion chromatography on Sephadex G-200, and in lacking blood group B-degrading activity. Fewer strains produced $\beta$-glucosidase, $\alpha$-mannosidase, and $\beta$-glucuronidase. Although each of the five mucin side chain degraders produced an H-degrading $\alpha$-L-fucosidase, this activity did not cleave $p$-nitrophenyl- $\alpha$-L-fucopyranoside. By contrast, all five Bacteroides strains produced a cell-bound $\alpha$-L-fucosidase that was active against the $p$-nitrophenyl substrate but not against $\mathrm{H}$ antigen. None of the seven activities were detected in cultures of the $E$. coli and $S$. faecalis strains.
Of the 21 strains, only two $S$. faecalis strains produced protease activity active on azoalbumin. This was exclusively extracellular and amounted to 180 and $500 \mathrm{U} / \mathrm{mg}$ bacterial protein, respectively.

\section{Discussion}

A distinguishing feature of the five strains of human fecal bacteria reported here is their ability to degrade oligosaccharide side chains of gut mucins. This characterizes them as a functionally unique and distinct subset of the normal human fecal flora. Thus, earlier work of Salyers et al. $(29,30)$ showed that none of 188 human fecal Bacteroides strains fermented HGM, and of 154 strains of 22 other human fecal anaerobic species only eight fermented HGM; these were six strains of $\boldsymbol{R}$. torques and two strains of Bifidobacterium bifidum. Our results provide independent confirmation that strains of $R$. torques and of bifidobacteria degrade mucin carbohydrates; fermentation of HGM with production of acids as measured by Salyers et al. appears to be equivalent to extensive degradation of mucin oligosaccharides as measured by us.

The basis for the ability of Bifidobacterium and Ruminococcus strains to degrade mucin oligosaccharides is their production of extracellular glycosidases with the appropriate linkage specificities for the glycosides comprising the chains. All produced sialidase and either one or two of the $\mathrm{ABH}$ blood groupdegrading $\alpha$-glycosidases that make the initial cleavage of the terminal $\alpha$-glycosides. Except for the lack of production of $\beta$ $N$-acetylhexosaminidases by $R . A B$ strain VI-268, these strains produced the extracellular $\beta$-glycosidases required for chain

Table VI. Production of Other Glycosidases by Fecal Isolates

\begin{tabular}{|c|c|c|c|c|c|}
\hline Glycosidase activity* & $\begin{array}{l}\text { Mucin side chain } \\
\text { degraders (5) }\end{array}$ & Bacteroides (5) & B. longum (1) & E. coli (5) & S. faecalis (5) \\
\hline pnp- $\alpha-N$-acetyl-D-glucosaminidase & $2 / 5$ & $0 / 5$ & $0 / 1$ & $0 / 5$ & $0 / 5$ \\
\hline pnp- $\alpha$-D-galactosidase & $3 / 5$ & $5 / 5$ & $1 / 1$ & $0 / 5$ & $0 / 5$ \\
\hline pnp- $\alpha$-D-glucosidase & $5 / 5$ & $5 / 5$ & $1 / 1$ & $0 / 5$ & $0 / 5$ \\
\hline pnp- $\beta$-D-glucosidase & $2 / 5$ & $3 / 5$ & $1 / 1$ & $0 / 5$ & $0 / 5$ \\
\hline pnp- $\alpha$-D-mannosidase & $1 / 5$ & $2 / 5$ & $0 / 1$ & $0 / 5$ & $0 / 5$ \\
\hline pnp- $\alpha$-L-fucosidase & $0 / 5$ & $5 / 5$ & $0 / 1$ & $0 / 5$ & $0 / 5$ \\
\hline$\beta$-D-glucuronidase & $1 / 5$ & $3 / 5$ & $0 / 1$ & $0 / 5$ & $0 / 5$ \\
\hline
\end{tabular}

* pnp, para-nitrophenyl-. Values, number of strains producing activity per number tested. 
degradation once the terminal $\alpha$-linked glycosides had been removed. By contrast, other fecal bacteria including the numerically abundant Bacteroides species did not produce blood group-degrading $\alpha$-glycosidases and produced others as cellbound enzymes. The limited ability of Bacteroides to degrade mucin oligosaccharides was also observed by Roberton and Stanley (31), who found that sialic acid residues were the major carbohydrate lost from pig gastric and colonic mucus during growth of $B$. fragilis in vitro.

The ability to produce $\alpha$-glycosidases with blood group A-, B-, or H-degrading activity is limited to only a few strains of human fecal bacteria. Previously reported fecal isolates with BGD activity are Clostridium perfringens $(27,32)$ (extracellular), Bifidobacterium bifidum var. Penn (33) (cell-bound), and Shigella dysenteriae (34) (cell bound). It is noteworthy that none of the strains reported here or previously cleaved all three blood group antigens. Each of our isolates produced $\mathrm{H}$ degrading activity alone or with either A- or B-degrading activity, supporting our earlier conclusion, based on differences in fecal population densities, that these enzyme activities were likely produced by separate bacterial populations (18). Since the $\mathrm{H}$ antigenic determinant $\alpha$-L-fucosyl residue is also present in the terminal trisaccharides of $A$ and $B$ antigens, the $H$ antigen residues could be removed by each of the strains, and the critical variable determining whether a given strain could continue the degradation of blood group A or B chains was its ability to produce the appropriate $\alpha$-glycosidase with A- or B-degrading specificity. A greater diversity of strains is thereby required for degradation of mucins containing blood group $A$ or B antigens than if a single strain produced glycosidases acting against all three blood group antigenic determinants. A competitive nutritional advantage should be conferred upon the strain that produces the $\alpha$-glycosidase that cleaves the blood group phenotype antigen from the host's mucin glycoproteins. The result should be an increased population density of that strain. This was the case for bacteria producing blood group B-degrading activity in B secretors (18) and is a presumed mechanism for the greater levels of B-degrading activity observed in fecal extracts (35) and whole fecal cultures (17) of blood group B secretors.

A noteworthy characteristic of glycosidase production by the five strains of oligosaccharide chain-degrading bacteria is that glycoside production was constitutive, i.e., did not require induction by mucin substrate. The ability to produce key nutritional enzymes constitutively has been shown to confer a selective advantage over nonconstitutive strains in chemostat cultures where substrate concentration is growth limiting (3638). A similar advantage might accrue to mucin oligosaccharide chain-degrading bacteria in their colonic habitat. In addition, there is an energy cost involved in producing glycosidases required for deriving nutritional monosaccharides from mucin oligosaccharides. It seems likely that less energy is expended within the colon ecosystem when the requisite glycosidases are produced constitutively as extracellular enzymes by a few specialized strains, thereby obviating the need for all resident strains to expend the energy required to produce them.

The extracellular glycosidases produced by the subset of fecal mucin oligosaccharide-degrading bacteria may serve several important biological roles. Experiments with whole fecal cultures indicate that monosaccharides released from mucin by these glycosidases support growth of larger populations of Bacteroides and other fecal bacterial that are otherwise unable to degrade and obtain benefit from mucins (39). Thus, mucindegrading strains may have a nutritional support role for human enteric bacteria, especially when the host is fasting. Their extracellular glycosidases are also capable of altering surface antigenic carbohydrates on bacterial cell walls (40) as well as on erythrocyte membranes (41). Comparable effects on membrane glycolipids and glycoproteins of the gut mucosa could lead to alterations of attachment sites for toxins and bacteria as well as damage to brush border enzymes in bacterial overgrowth syndromes (42). These glycosidases may also contribute to the activation of mutagens by deconjugation of ingested plant glycosides $(43,44)$. Their role in these activities as well as in ulcerative lesions of the gut mucosa remains to be determined.

\section{Appendix}

Calculation of the probabilities that glycosidases occurring together in cultures are produced by a single bacterial strain (hypothesis I) or by separate bacterial strains, each producing a single glycosidase (hypothesis II). Let there be $n$ replicate cultures inoculated from a single fecal dilution where glycosidase activities required for mucin side chain degradation occur together in $r$ cultures. Extensive side chain degradation occurs in these cultures and not in $(n-r)$ cultures. Assume that bacteria are randomly and independently distributed throughout all cultures. Glycosidases A, B, C, D, . . a are detected among the $n$ cultures. Glycosidase $\mathrm{A}$ is present in $a$ cultures, $\mathrm{B}$ is present in $b$ cultures, $\mathrm{C}$ is present in $c$ cultures, $\mathrm{D}$ is present in $d$ cultures, etc. Glycosidases A, B, C, D, etc., occur together in $r$ cultures. The size of the fecal inoculum is such that $n>a, b, c, d, \ldots r$.

Hypothesis I. All glycosidases are produced by a single bacterial strain which is present in $r$ cultures. The probability that any one culture contains all of the glycosidases equals $r / n$. Consider that $n$ replicate cultures were drawn at random from a large number of cultures, each with the probability $r / n$ of containing the glycosidases. Then the probability of obtaining all glycosidases together in exactly $r$ cultures under hypothesis $I$ is:

$\mathrm{P}_{(\mathrm{H}-\mathrm{l})}={ }_{n} \mathrm{C}_{r}(r / n)^{r}(1-r / n)^{n-r}$,

where ${ }_{n} \mathrm{C}_{r}$ equals the number of combinations of $n$ things taken $r$ at a time equals $n ! / r !(n-r) !$.

Hypothesis II. Each of the glycosidases is produced by a separate bacterial strain. Strain A produces glycosidase A, strain B produces B, strain $C$ produces $C$, and strain $D$ produces $D$, etc. The probability that any one culture contains glycosidase $\mathrm{A}$ is $\mathrm{P}_{\mathrm{A}}=a / n$, glycosidase $\mathrm{B}$ is $\mathrm{P}_{\mathrm{B}}=b / n$, etc. The probability that any one culture contains all glycosidases is $a / n \cdot b / n \cdot c / n \cdot d / n=\mathrm{P}_{\mathrm{A}} \cdot \mathrm{P}_{\mathrm{B}} \cdot \mathrm{P}_{\mathrm{C}} \cdot \mathrm{P}_{\mathrm{D}}=\prod_{i=\mathrm{A}}^{\mathrm{D}} \mathrm{P}_{i}$. Consider that $n$ cultures were drawn at random from a large number of cultures, each with the probability $\prod_{i=\mathrm{A}}^{\mathrm{D}} \mathrm{P}_{i}$ of containing all the glycosidases. Then the probability of obtaining all the glycosidases together in exactly $r$ cultures under hypothesis II is:

$\mathrm{P}_{(\mathrm{H}-\mathrm{II})}={ }_{n} \mathrm{C}_{r}\left(\prod \mathrm{P}_{i}\right)^{r}\left(1-\prod \mathrm{P}_{i}\right)^{n-r}$,

and the odds favoring hypothesis $\mathrm{I}=\mathrm{P}_{(\mathrm{H}-\mathrm{I})} / \mathrm{P}_{(\mathrm{H}-\mathrm{II})}$.

The following four requirements must be met for application of binominal probability measurements to a sampling design (45): (a) there must be a fixed number of trials, $n ;(b)$ the probability of success must be the same in each trial; $(c)$ the trials must be independent of each other; and $(d)$ there must be either one of two mutually exclusive outcomes in each trial. In the present application each one of $n$ replicate cultures is regarded as a separate and independent trial where the probability of "success", i.e., $r / n, \Pi \mathrm{P}_{i}$, is the same for each culture. These conditions are met if bacteria are randomly and 
independently distributed throughout all cultures. This was approximated by thorough mixing during the serial diluting and sampling procedures. The two mutually exclusive outcomes were the presence or absence of each glycosidase activity in each culture. As noted in Methods, the glycosidase activities met this requirement through choice of assay conditions or use of discriminating cut-off values.

Note added in proof. Three of the mucin oligosaccharide-degrading strains have been deposited in The American Type Culture Collection. These are $R$. $A B$ strain VI-268 (ATCC No. 35913), B. bifidum strain VIII-210 (ATCC No. 35914), and $R$. torques strain IX-70 (ATCC No. 35915).

\section{Acknowledgments}

We thank Dr. Lillian V. Holdeman and the staff of the Anaerobe Laboratory, Virginia Polytechnic Institute, for establishing the taxonomic identity of the five mucin oligosaccharide chain-degrading strains, and Dr. Paul K. Jones of the Department of Biometry for helpful advice about the probability equations.

This work was supported by a research grant from the Veterans Administration. Dr. McKee was supported by a summer research grant from the Cleveland branch of The English Speaking Union and for a second summer by a Student Research Fellowship of The American Gastroenterological Association.

\section{References}

1. Allen, A., A. Bell, M. Mantle, and J. P. Pearson. 1982. The structure and physiology of gastrointestinal mucus. Mucus in health and disease. II. Adv. Exp. Med. Biol. 144:115-133.

2. Allen, A., and A. Garner. 1980. Mucus and bicarbonate secretion in the stomach and their possible role in mucosal protection. Gut. 21: 249-262.

3. Rozee, K. R., D. Cooper, K. Lam, and J. W. Costerton. 1982. Microbial flora of the mouse ileum mucous layer and epithelial surface. Appl. Environ. Microbiol. 43:1451-1463.

4. Cohen, P. S., R. Rossoll, V. J. Cabelli, S. L. Yang, and D. L. Laux. 1983. Relationship between the mouse colonizing ability of a human fecal Escherichia coli strain and its ability to bind a specific mouse colonic mucous gel protein. Infect. Immun. 40:62-69.

5. Freter, R. 1982. Bacterial association with the mucus gel system of the gut. In Microbiology. D. Schlessinger, editor. American Society of Microbiology, Washington, DC. 278-281.

6. Silberberg, A., and F. A. Meyer. 1982. Structure and function of mucus. Mucus in health and disease. II. Adv. Exp. Med. Biol. 144: 53-74.

7. Van Halbeek, H., L. Dorland, J. F. G. Valiegenthart, N. K. Kochetkov, N. P. Arbatsky, and V. A. Derevitskaya. 1982. Characterization of the primary structure and microheterogeneity of the carbohydrate chains of porcine blood-group $\mathrm{H}$ substance by $500 \mathrm{MHz}{ }^{1} \mathrm{H}$ NMR spectroscopy. Eur. J. Biochem. 127:21-29.

8. Derevitskaya, V. A., N. P. Arbatsky, and K. K. Kochetkov. 1978. The structure of carbohydrate chains of blood-group substance. Isolation and elucidation of the structure of higher oligosaccharides from blood group substance H. Eur. J. Biochem. 86:423-437.

9. Slomiany, B. L., E. Zdebska, and A. Slomiany. 1984. Structural characterization of neutral oligosaccharides of human $\mathrm{H}^{+} \mathrm{Le}^{\mathrm{b}}$ gastric mucin. J. Biol. Chem. 259:2863-2869.

10. Slomiany, A., E. Zdebska, and B. L. Slomiany. 1984. Structure of the neutral oligosaccharides isolated from A-active human gastric mucin. J. Biol. Chem. 259:14743-14749.

11. Lindstedt, G., S. Lindstedt, and B. E. Gustafsson. 1965. Mucus in intestinal contents of germfree rats. J. Exp. Med. 121:201-213.

12. Hoskins, L. C., and N. Zamchek. 1968. Bacterial degradation of gastrointestinal mucins. 1. Comparison of mucus constituents in the stools of germ-free and conventional rats. Gastroenterology. 54: 210-217.
13. Vercellotti, J. R., A. A. Salyers, W. S. Bullard, and T. D. Wilkins. 1977. Breakdown of mucin and plant polysaccharides in the human colon. Can. J. Biochem. 55:1190-1196.

14. Variyam, E. P., and L. C. Hoskins. 1981. Mucin degradation in human colon ecosystems. Degradation of hog gastric mucin by fecal extracts and fecal cultures. Gastroenterology. 81:751-758.

15. Miller, R. S., and L. C. Hoskins. 1981. Mucin degradation in human colon ecosystems. Fecal population densities of mucin-degrading bacteria estimated by a "most probable number" method. Gastroenterology. 81:759-765.

16. Hoskins, L. C., and E. T. Boulding. 1981. Mucin degradation in human colon ecosystems. Evidence for the existence and role of bacterial subpopulations producing glycosidases as extracellular enzymes. J. Clin. Invest. 67:163-172.

17. Hoskins, L. C., and E. T. Boulding. 1976. Degradation of blood group antigens in human colon escosystems. I. In vitro production of $\mathrm{ABH}$ blood group-degrading enzymes by fecal bacteria. J. Clin. Invest. 57:63-73.

18. Hoskins, L. C., and E. T. Boulding. 1976. Degradation of blood group antigens in human colon ecosystems. II. A gene interaction in man that affects the fecal population density of certain enteric bacteria. J. Clin. Invest. 57:74-82.

19. Kabat, E. A., H. Baer, A. E. Bezer, and V. Knaub. 1948. Immunochemical studies on blood groups. VII. Chemical changes associated with destruction of blood group activity and enhancement of the type XIV cross-reactivity by partial hydrolysis of hog and human blood group A, B, and O substances. J. Exp. Med. 88:43-57.

20. Holdeman, L. V., E. P. Cato, and W. E. C. Moore. 1977. Anaerobe Laboratory Manual. Virginia Polytechnic Institute Anaerobe Laboratory, Blacksburg, VA. Fourth ed. 152 pp.

21. Meynell, G. G., and E. Meynell. 1970. Theory and Practice in Experimental Microbiology. Cambridge University Press, New York. Second ed. $347 \mathrm{pp}$.

22. Moore, W. E. C., and L. V. Holdeman. 1974. Human fecal flora. The normal flora of 20 Japanese-Hawaiians. Appl. Microbiol. 27: 961-979.

23. Talalay, P., W. H. Fishman, and C. Huggins. 1946. Chromogenic substrates. II. Phenolphthalein glucuronic acid as substrate for the assay of glucuronidase activity. J. Biol. Chem. 166:757-772.

24. Tomarelli, R. M., J. Charney, and M. L. Harding. 1949. The use of azoalbumin as a substrate in the colorimetric determination of peptic and tryptic activity. J. Lab. Clin. Med. 34:428-433.

25. Reissig, J. E., J. L. Strominger, and L. F. Leloir. 1955. A modified colorimetric method for the estimation of $\mathrm{N}$-acetylamino sugars. J. Biol. Chem. 217:959-966.

26. Lowry, O. H., N. J. Rosebrough, A. J. Farr, and R. J. Randall. 1951. Protein measurement with the folin phenol reagent. J. Biol. Chem. 193:265-275.

27. Levy, G. N., and D. Aminoff. 1980. Purification and properties of $\alpha-\mathrm{N}$-acetylgalactosaminidase from Clostridium perfringens. J. Biol. Chem. 255:11737-11742.

28. Richmond, M. H. 1968. Enzymic adaptation in bacteria: its biochemical and genetic basis. Essays Biochem. 4:105-154.

29. Salyers, A. A., J. R. Vercellotti, S. E. H. West, and T. D. Wilkins. 1977. Fermentation of mucin and plant polysaccharides by strains of Bacteroides from the human colon. Appl. Environ. Microbiol. 33:319-322.

30. Salyers, A. A., S. E. H. West, J. R. Vercellotti, and T. D. Wilkins. 1977. Fermentation of mucin and plant polysaccharides by anaerobic bacteria from the human colon. Appl. Environ. Microbiol. 34:529-533.

31. Roberton, A. M., and R. A. Stanley. 1982. In vitro utilization of mucin by Bacteroides fragilis. Appl. Environ. Microbiol. 43:325330.

32. Aminoff, D., and K. Furukawa. 1970. Enzymes that destroy blood group specificity. I. Purification and properties of $\alpha$-L-fucosidase from Clostridium perfringens. J. Biol. Chem. 245:1659-1669.

33. Gyorgy, P., C. S. Rose, and G. F. Springer. 1954. Enzymatic 
inactivation of Bifidus Factor and blood group substances. J. Lab. Clin. Med. 43:543-552.

34. Prizont, R. 1982. Degradation of intestinal glycoproteins by pathogenic Shigella flexneri. Infect. Immun. 36:615-620.

35. Hoskins, L. C. 1969. Ecological studies of intestinal bacteria. Relation between the specificity of fecal $\mathrm{ABO}$ blood group antigendegrading enzymes from enteric bacteria and the $\mathrm{ABO}$ blood group of the human host. J. Clin. Invest. 48:664-673.

36. Dykhuizen, D. E., and D. L. Hartl. 1982. Selection in chemostats. Microbiol. Rev. 47:150-168.

37. Dykhuizen, D., and M. Davies. 1980. An experimental model: bacterial specialists and generalists competing in chemostats. Ecology. 61:1213-1227.

38. Horiuchi, T., J. I. Tomizawa, and A. Novick. 1962. Isolation and properties of bacteria capable of high rates of $\beta$-galactosidase synthesis. Biochim. Biophys. Acta. 55:152-163.

39. Harouny, V. R., and L. C. Hoskins. 1983. A role for mucindegrading bacteria in nutritional support of human enteric bacteria. Gastroenterology. 84:1405. (Abstr.)
40. Cromwell, C. L., and L. C. Hoskins. 1977. Antigen degradation in human colon ecosystems. Host's ABO blood type influences enteric bacterial degradation of a cell surface antigen on Escherichia coli 086. Gastroenterology. 73:37-41.

41. Hoskins, L. C. 1984. Mucin degradation by enteric bacteria: ecological aspects and implications for bacterial attachment to gut mucosa. In Attachment of Organisms to the Gut Mucosa. E. C. Boedeker, editor. CRC Press, Boca Raton, FL. II:51-67.

42. Prizont, R. 1981. Glycoprotein degradation in the blind loop syndrome. J. Clin. Invest. 67:336-344.

43. Brown, J. P., and P. S. Dietrich. 1979. Mutagenicity of plant flavonols in the Salmonella/mammalian microsome test. Activation of flavonol glycosides by mixed glycosidases from rat cecal bacteria and other sources. Mutat. Res. 66:223-240.

44. Tamura, G., C. Gold, A. Ferro-Luzzi, and B. N. Ames. 1980. Fecalase: a model for activation of dietary glycosides and mutagens by intestinal flora. Proc. Natl. Acad. Sci. USA. 77:4961-4965.

45. Mosteller, E., R. E. K. Rourke, and G. B. Thomas, Jr. 1961. Probability with Statistical Applications. Addison-Wesley, Reading, MA. $478 \mathrm{pp}$. 